PROCEEDINGS OF THE

AMERICAN MATHEMATICAL SOCIETY

Volume 129, Number 9, Pages 2547-2552

S 0002-9939(01)05922-6

Article electronically published on January 23, 2001

\title{
COMPARISON OF 4-CLASS RANKS OF CERTAIN QUADRATIC FIELDS
}

\author{
FRANK GERTH III
}

(Communicated by David E. Rohrlich)

\begin{abstract}
Let $m$ be a square-free positive integer. Let $r_{4}(K)$ denote the 4-class rank of a quadratic field $K$. This paper examines how likely it is for $r_{4}(\mathbb{Q}(\sqrt{-m}))=r_{4}(\mathbb{Q}(\sqrt{m}))$ and for $r_{4}(\mathbb{Q}(\sqrt{-m}))=r_{4}(\mathbb{Q}(\sqrt{m}))+1$.
\end{abstract}

\section{IntRoduction}

Let $K$ be a quadratic extension of the field of rational numbers $\mathbb{Q}$. Let $C(K)$ denote the 2-class group of $K$ in the narrow sense. It is well known that $\operatorname{rank} C(K)=$ $t-1$, where $t$ is the number of primes that ramify in $K / \mathbb{Q}$. Let $r_{4}(K)$ denote the 4 -class rank of $K$ in the narrow sense; i.e.,

$$
r_{4}(K)=\operatorname{dim}_{\mathbb{F}_{2}}\left((C(K))^{2} /(C(K))^{4}\right)
$$

where $(C(K))^{i}=\left\{c^{i}: c \in C(K)\right\}$ for positive integers $i$, and $\mathbb{F}_{2}$ is the finite field with two elements. In Equation (1.1), we are viewing the elementary abelian 2-group $(C(K))^{2} /(C(K))^{4}$ as a vector space over $\mathbb{F}_{2}$.

Now let $m$ be a square-free positive integer. It is known (cf. [2], 6]) that

$$
r_{4}(\mathbb{Q}(\sqrt{m})) \leq r_{4}(\mathbb{Q}(\sqrt{-m})) \leq r_{4}(\mathbb{Q}(\sqrt{m}))+1 .
$$

We will consider the following question: how likely is it that $r_{4}(\mathbb{Q}(\sqrt{-m}))=$ $r_{4}(\mathbb{Q}(\sqrt{m}))$, and how likely is it that $r_{4}(\mathbb{Q}(\sqrt{-m}))=r_{4}(\mathbb{Q}(\sqrt{m}))+1$ ? A direct answer could be obtained if we could compute

$$
\lim _{x \rightarrow \infty} \frac{\mid\left\{\text { square-free } m \leq x: r_{4}(\mathbb{Q}(\sqrt{-m}))=r_{4}(\mathbb{Q}(\sqrt{m}))\right\} \mid}{\mid\{\text { square-free } m \leq x\} \mid}
$$

or

$$
\lim _{x \rightarrow \infty} \frac{\mid\left\{\text { square-free } m \leq x: r_{4}(\mathbb{Q}(\sqrt{-m}))=r_{4}(\mathbb{Q}(\sqrt{m}))+1\right\} \mid}{\mid\{\text { square-free } m \leq x\} \mid}
$$

where $|S|$ denotes the cardinality of a set $S$. However, computing these limits appears to be very difficult. Instead, we shall use a somewhat different approach. Although the limits we compute are not guaranteed to equal the above limits, our results do provide some insight into this question.

Received by the editors January 19, 2000.

2000 Mathematics Subject Classification. Primary 11R11, 11R29, 11R45.

(C)2001 American Mathematical Society 
First we introduce some notation. For positive integers $t$, nonnegative integers $i$, positive real numbers $x$, and square-free positive integers $m$, we define

$$
\begin{aligned}
A_{t ; x} & =\{\mathbb{Q}(\sqrt{-m}): \text { exactly } t \text { primes ramify in } \mathbb{Q}(\sqrt{-m}) / \mathbb{Q} \text { and } m \leq x\}, \\
A_{t, i ; x} & =\left\{\mathbb{Q}(\sqrt{-m}) \in A_{t ; x}: r_{4}(\mathbb{Q}(\sqrt{-m}))=i\right\}, \\
A_{t, i ; x}^{(1)} & =\left\{\mathbb{Q}(\sqrt{-m}) \in A_{t, i ; x}: r_{4}(\mathbb{Q}(\sqrt{-m}))=r_{4}(\mathbb{Q}(\sqrt{m}))\right\}, \\
A_{t, i ; x}^{(2)} & =\left\{\mathbb{Q}(\sqrt{-m}) \in A_{t, i ; x}: r_{4}(\mathbb{Q}(\sqrt{-m}))=r_{4}(\mathbb{Q}(\sqrt{m}))+1\right\} .
\end{aligned}
$$

We then define the following densities:

$$
\begin{aligned}
& a_{t, i}=\lim _{x \rightarrow \infty} \frac{\left|A_{t, i ; x}\right|}{\left|A_{t ; x}\right|}, \\
& a_{t, i}^{(1)}=\lim _{x \rightarrow \infty} \frac{\left|A_{t, i ; x}^{(1)}\right|}{\left|A_{t ; x}\right|}, \\
& a_{t, i}^{(2)}=\lim _{x \rightarrow \infty} \frac{\left|A_{t, i ; x}^{(2)}\right|}{\left|A_{t ; x}\right|} .
\end{aligned}
$$

Next we define the limit densities:

$$
\begin{aligned}
& a_{\infty, i}=\lim _{t \rightarrow \infty} a_{t, i}, \\
& a_{\infty, i}^{(1)}=\lim _{t \rightarrow \infty} a_{t, i}^{(1)}, \\
& a_{\infty, i}^{(2)}=\lim _{t \rightarrow \infty} a_{t, i}^{(2)} .
\end{aligned}
$$

It is known (cf. Equation (1.5) in [4]) that

$$
a_{\infty, i}=\frac{2^{-i^{2}} \prod_{k=1}^{\infty}\left(1-2^{-k}\right)}{\prod_{k=1}^{i}\left(1-2^{-k}\right)^{2}}
$$

for $i=0,1,2, \cdots$. Furthermore, $\sum_{i=0}^{\infty} a_{\infty, i}=1$, and, of course, $a_{\infty, i}=a_{\infty, i}^{(1)}+a_{\infty, i}^{(2)}$. To obtain the likelihood that $r_{4}(\mathbb{Q}(\sqrt{-m}))=r_{4}(\mathbb{Q}(\sqrt{m}))$ and that $r_{4}(\mathbb{Q}(\sqrt{-m}))=$ $r_{4}(\mathbb{Q}(\sqrt{m}))+1$, we let

$$
\begin{aligned}
& \alpha_{1}=\sum_{i=0}^{\infty} a_{\infty, i}^{(1)}, \\
& \alpha_{2}=\sum_{i=0}^{\infty} a_{\infty, i}^{(2)} .
\end{aligned}
$$

We shall prove the following theorem. 
Theorem 1. Let $\alpha_{1}$ and $\alpha_{2}$ be defined by Equations (1.10) and (1.11), and let $a_{\infty, i}$ be given by Equation (1.9). Then

$$
\begin{aligned}
& \alpha_{1}=\sum_{i=0}^{\infty} 2^{-i} a_{\infty, i} \approx 0.610321 ; \\
& \alpha_{2}=\sum_{i=0}^{\infty}\left(1-2^{-i}\right) a_{\infty, i} \approx 0.389679 .
\end{aligned}
$$

Remark. Theorem 1 is also valid if we use the 4 -class rank in the usual sense rather than the narrow sense (cf. discussion on p. 491 of [4]).

\section{Proof of Theorem 1}

From the discussion on p. 491 of [4, it suffices to consider $m=p_{1} \cdots p_{t}$ with distinct odd primes $p_{1}, \cdots, p_{t}$ and with an odd number of primes $p_{i} \equiv 3(\bmod 4)$ when analyzing $A_{t ; x}$ and its subsets in our counting arguments. For convenience we label the primes so that

$$
\begin{aligned}
& p_{i} \equiv 1 \quad(\bmod 4) \text { for } 1 \leq i \leq s, \\
& p_{i} \equiv 3 \quad(\bmod 4) \text { for } s+1 \leq i \leq t
\end{aligned}
$$

where $s \geq 0$ and $t-s$ is odd. Now the 4 -class rank of $K=\mathbb{Q}(\sqrt{-m})$ satisfies

$$
r_{4}(K)=t-1-\operatorname{rank} M_{K}^{\prime}
$$

where $M_{K}^{\prime}=\left[b_{i j}\right]$ is the $t \times(t-1)$ matrix with entries in $\mathbb{F}_{2}$ defined by Legendre symbols as follows:

$$
(-1)^{b_{i j}}= \begin{cases}\left(\frac{P_{j}}{p_{i}}\right), & \text { if } i \neq j, \\ \left(\frac{-m / P_{j}}{p_{i}}\right), & \text { if } i=j,\end{cases}
$$

for $1 \leq i \leq t$ and $1 \leq j \leq t-1$ (cf. Equation (2.6) in [4]). Here $P_{j}=p_{j}$ if $p_{j} \equiv 1$ $(\bmod 4)$, and $P_{j}=-p_{j}$ if $p_{j} \equiv 3(\bmod 4)$. As discussed on p. 492 in 4], it is also true that

$$
r_{4}(K)=t-1-\operatorname{rank} M_{K}
$$

where $M_{K}$ is the $t \times t$ matrix with entries defined by Equation (2.4), except with $1 \leq j \leq t$ instead of $1 \leq j \leq t-1$. Furthermore, the sum of the entries in each row of $M_{K}$ is zero, and the sum of the entries in each column of $M_{K}$ is zero.

Now we let $L=\mathbb{Q}(\sqrt{m})$. Since there are an odd number of primes $p_{i} \equiv 3$ $(\bmod 4)$ that divide $m$, then $m \equiv 3(\bmod 4)$. So $t+1$ primes ramify in $L / \mathbb{Q}$; namely $p_{1}, \cdots, p_{t}$ and 2 . The 4 -class rank of $L$ satisfies

$$
r_{4}(L)=(t+1)-1-\operatorname{rank} M_{L}^{\prime}
$$

where $M_{L}^{\prime}=\left[c_{i j}\right]$ is the $(t+1) \times t$ matrix over $\mathbb{F}_{2}$ whose entries satisfy

$$
c_{i j}=\left\{\begin{array}{l}
b_{i j} \text { if }(i \neq j \text { and } 1 \leq i \leq t, 1 \leq j \leq t) \text { or if }(i=j \text { and } 1 \leq i \leq s), \\
b_{i j}+1 \text { if } i=j \text { and } s+1 \leq i \leq t \\
0 \text { if } i=t+1 \text { and } 2 \text { splits in } \mathbb{Q}\left(\sqrt{P_{j}}\right), \\
1 \text { if } i=t+1 \text { and } 2 \text { remains prime in } \mathbb{Q}\left(\sqrt{P_{j}}\right) .
\end{array}\right.
$$


Let $M_{L}$ denote the $t \times t$ matrix consisting of the first $t$ rows of $M_{L}^{\prime}$.

Lemma 1. Rank $M_{L}=\operatorname{rank} M_{K}+1$.

Proof. Write

$$
M_{K}=\left[\begin{array}{c:c}
B_{1} & B_{2} \\
\hdashline B_{3} & B_{4}
\end{array}\right]
$$

where $B_{1}$ is an $s \times s$ symmetric matrix over $\mathbb{F}_{2}, B_{2}$ is an $s \times(t-s)$ matrix over $\mathbb{F}_{2}, B_{3}$ is the $(t-s) \times s$ matrix which equals $B_{2}^{T}$ (the transpose of $B_{2}$ ), and $B_{4}$ is a $(t-s) \times(t-s)$ antisymmetric matrix over $\mathbb{F}_{2}$ (i.e., $b_{i j}=b_{j i}+1$ for $i \neq j$ ). These properties follow from Equation (2.4) and quadratic reciprocity. Note that

$$
M_{K}^{T}=\left[\begin{array}{c:c}
B_{1}^{T} & B_{3}^{T} \\
\hdashline B_{2}^{T} & B_{4}^{T}
\end{array}\right]=\left[\begin{array}{c:c}
B_{1} & B_{2} \\
\hdashline B_{3} & B_{4}+I+J
\end{array}\right]
$$

where $I$ is the $(t-s) \times(t-s)$ identity matrix, and $J$ is the $(t-s) \times(t-s)$ matrix with each entry equal to 1 . Now from Equations (2.7) and (2.8) and our definition of $M_{L}$,

$$
M_{L}=\left[\begin{array}{c:c}
B_{1} & B_{2} \\
\hdashline B_{3} & B_{4}+I
\end{array}\right]=\left[\begin{array}{c:c}
B_{1} & B_{2} \\
\hdashline B_{3} & B_{4}+I+2 J
\end{array}\right]=M_{K}^{T}+H
$$

since $2 J$ is a zero matrix over $\mathbb{F}_{2}$, and where

$$
H=\left[\begin{array}{c:c}
O & O \\
\hdashline O & J
\end{array}\right] \text {. }
$$

Now let $v \in \mathbb{F}_{2}^{t}$. (Think of $v$ as a column vector.) If the last $(t-s)$ entries in $v$ contain an even number of 1 's, then

$$
M_{L} v=M_{K}^{T} v+H v=M_{K}^{T} v .
$$

Let

$W=\left\{M_{K}^{T} v: v \in \mathbb{F}_{2}^{t}\right.$ with an even number of 1's in the last $(t-s)$ entries in $\left.v\right\}$.

If the last $(t-s)$ entries in $v$ contain an odd number of 1 's, let $v_{1}=v+v_{2}$, where $v_{2}=[0, \cdots, 0,1]^{T}$. Note that the last $(t-s)$ entries in $v_{1}$ contain an even number of 1 's, and $v=v_{1}+v_{2}$. Then

$$
M_{K}^{T} v=M_{K}^{T} v_{1}+M_{K}^{T} v_{2} .
$$

Clearly $M_{K}^{T} v_{1} \in W$. Next, note that $M_{K}^{T} v_{2}=M_{K}^{T} v_{3}$, where $v_{3}=[1, \cdots, 1,0]^{T}$, since the sum of the entries in each row of $M_{K}^{T}$ is zero. But then $M_{K}^{T} v_{3} \in W$ since $v_{3}$ has an even number of 1 's in its last $(t-s)$ entries. Thus

$$
M_{K}^{T} v=M_{K}^{T} v_{1}+M_{K}^{T} v_{3} \in W .
$$

So $W$ is the column space of $M_{K}^{T}$, and from Equation (2.11), we know that the column space of $M_{L}$ contains $W$. Also, since the matrix $H$ in Equation (2.10) has rank equal to 1, then from Equation (2.9), we know that

$$
\operatorname{rank} M_{K}^{T} \leq \operatorname{rank} M_{L} \leq \operatorname{rank} M_{K}^{T}+1 .
$$

Now let $v_{4}$ be the vector in $\mathbb{F}_{2}^{t}$ with each component equal to 1 . Then $M_{K}^{T} v_{4}$ is the zero vector in $\mathbb{F}_{2}^{t}$ since the sum of the entries in each row of $M_{K}^{T}$ is zero. Then from 
Equations (2.9) and (2.10), $M_{L} v_{4}$ is the vector in $\mathbb{F}_{2}^{t}$ whose first $s$ components are 0 's and whose last $(t-s)$ components are 1's since $t-s$ is odd. Then the sum of the entries in $M_{L} v_{4}$ is 1 . But then $M_{L} v_{4}$ does not belong to the column space of $M_{K}^{T}$ since the sum of the entries in each column of $M_{K}^{T}$ is zero. Thus from (2.13), we see that

$$
\operatorname{rank} M_{L}=\operatorname{rank} M_{K}^{T}+1=\operatorname{rank} M_{K}+1
$$

which completes the proof of Lemma 1.

We let

$$
w=\operatorname{rank} M_{K}=\operatorname{rank} M_{L}-1 .
$$

We now consider the $(t+1) \times t$ matrix $M_{L}^{\prime}$ whose first $t$ rows form the matrix $M_{L}$. From Equation (2.7), we observe that the entries in the last row of $M_{L}^{\prime}$ satisfy

$$
c_{(t+1) j}=\left\{\begin{array}{lll}
0, & \text { if } p_{j} \equiv \pm 1 \quad(\bmod 8) \\
1, & \text { if } p_{j} \equiv \pm 3 \quad(\bmod 8)
\end{array}\right.
$$

Since the primes are equally distributed among the residue classes $\pm 1(\bmod 8)$ and $\pm 3(\bmod 8)$, it is intuitively clear that each entry in the last row of $M_{L}^{\prime}$ is equally likely to be a 0 or a 1 . (This can be proved using character sums similar to those used to prove Propositions 2.1 and 5.1 in [4]. See [3] and [5] (or [1]) for more details on character sum calculations.) Then, of the possible $2^{t}$ matrices $M_{L}^{\prime}$ whose first $t$ rows form $M_{L}, 2^{1+w}$ satisfy rank $M_{L}^{\prime}=\operatorname{rank} M_{L}$, and $\left(2^{t}-2^{1+w}\right)$ satisfy $\operatorname{rank} M_{L}^{\prime}=\operatorname{rank} M_{L}+1$. From Equation (2.6) and the above discussion,

$$
r_{4}(L)= \begin{cases}t-1-w & \text { with probability } 2^{-(t-1-w)} \\ t-2-w & \text { with probability } 1-2^{-(t-1-w)} .\end{cases}
$$

Then Equations 2.5, 2.14, and 2.15 give

$$
r_{4}(K)= \begin{cases}r_{4}(L) & \text { with probability } 2^{-(t-1-w)} \\ r_{4}(L)+1 & \text { with probability } 1-2^{-(t-1-w)} .\end{cases}
$$

Then letting $i=t-1-w$ and using Equations 1.3, 1.4, and 1.5, we get

$$
a_{t, i}^{(1)}=2^{-i} a_{t, i} \text { and } a_{t, i}^{(2)}=\left(1-2^{-i}\right) a_{t, i} .
$$

Taking the limit as $t \rightarrow \infty$, we get

$$
a_{\infty, i}^{(1)}=2^{-i} a_{\infty, i} \text { and } a_{\infty, i}^{(2)}=\left(1-2^{-i}\right) a_{\infty, i} .
$$

Then summing over all $i \geq 0$, we get Theorem 1 .

\section{REFERENCES}

1. J. Cremona and R. Odoni, Some density results for negative Pell equations; an application of graph theory, J. London Math. Soc. 39 (1989), 16-28. MR 90b:11019

2. P. Damey and J. Payan, Existence et construction des extensions galoisiennes et nonabéliennes de degré 8 d'un corps de caractéristique différente de 2, J. Reine Angew. Math. 244 (1970), 37-54. MR 43:6186

3. F. Gerth, Counting certain number fields with prescribed $\ell$-class numbers, J. Reine Angew. Math. 337 (1982), 195-207. MR 84c:12002

4. F. Gerth, The 4-class ranks of quadratic fields, Invent. Math. 77 (1984), 489-515. MR 85j: 11137 
5. F. Gerth, Densities for ranks of certain parts of p-class groups, Proc. Amer. Math. Soc. 99 (1987), 1-8. MR 88b:11067

6. F. Halter-Koch, Über den 4-Rank der Klassengruppe quadratischer Zahlkörper, J. Number Theory 19 (1984), 219-227. MR 86a:11041

Department of Mathematics, The University of Texas at Austin, Austin, Texas 787121082

E-mail address: gerth@math.utexas.edu 